Slaviša Sovilj ${ }^{1}$, Vojkan Vasković ${ }^{2}$

${ }^{1}$ Ministry of Internal Affairs of the Republic of Srpska, Bosnia and Herzegovina 2 University of Belgrade, Faculty of Organizational Sciences, the Republic of Serbia

\title{
Exploring the Usefulness of Corporate Online Social Networks in the Human Resource Management
}

UDC: $005.96: 005.57 ; 316.472 .4: 004.738 .5$

DOI: 10.7595/management.fon.2014.0002

\begin{abstract}
Finding and selecting the appropriate personnel in work organizations has always been a problem. Companies need to exploit the potential of their employees to take advantage of their knowledge, connections and contacts, to engage in new projects and jobs or to move them to other positions. In doing their jobs employees establish contacts within the company and the business environment. Until the advent of the Internet, communication was carried out in a conventional manner and business did not allow to simply follow the links and communication between employees and their relationships with the business environment. Today, as part of e-business, employees communicate with each other, send and receive messages, simply put, create a social network. The analysis of these networks provides a wealth of information that can be used to promote business, find employees who represent the nodes of communication, but also provides a wealth of information on employees or those who are interested in the right jobs, who use social networks to post information about themselves.

This paper explored the possibility of obtaining information relevant to the selection of internal human resources based on an analysis of corporate online social networks. Research methods are taken from the field of graph theory and social network analysis (SNA), whereas in addition to quantitative parameters of nodes also additional dimensions of data filtering are considered. This approach is called the extended SNA. In addition to demonstrating and explaining, the extended SNA has developed an application that simulates the communication between employees within a corporation, for the analysis and detection of suitable employees, and visualizes the results in the form of a graph.
\end{abstract}

Keywords: Corporate social network, human resources, social network analysis, simulation

\section{Introduction}

Human resource management is a strategic position in the company, which is aimed at planning, managing, and scheduling of employees within the corporation. Activities of human resource planning determine the actual need of employees and they can be provided both from an external supply on the labor market or from existing resources. The process of planning the required staff begins by identifying the jobs for which executives are needed. Filling of vacancies can be provided through the analysis of internal supply and selection from the existing human resources or by hiring labour from the labor market. Depending on the activities of the corporation, the analysis of internal human resources can be done by exploring the interaction of employees in the corporate online social network. The online corporate social network (OKSM) is an information and communication platform that includes a variety of communication channels based on telephony, Web 2.0, instant messaging and e - mail technology.

\section{Corporate social network - enterprise 2.0 concept}

The Web 2.0 implies the evolution of a passive viewing of content and the possibility of generating and sharing the content on the Web. With the advent of interactive Web comes the creating of online social cohesion user, through the application of social networking. The SixDegrees.com [4] was the first example of social net- 
working as we know it today. This application is worked out based on user profiles similar to what is currently done with Facebook and was active in the period from 1997 to 2001. The term social network refers to a connection between members of the network, while in the virtual environment it refers to the use of the existing tools for communication, collaboration, interaction and sharing of ideas, interests and knowledge [4].

Examples of virtual social networks are profiled applications, communities, instant messaging, blogs and wikis. What is unique about these networks is that the social connections of the participants are easily visible, while social interactions in the real world are hardly visible and inaccessible for measurement [18]. Also, social networks participants set available personal information, which significantly facilitates a social research to be conducted. The concept of social networking is mapped to a network of employees in a company called [15], Enterprise 2.0 refers to the use of social application platforms within companies ( E2E ), business partners ( B2B) and customer ( B2C ) with the aim of better collaboration and information sharing in an enterprise environment. In terms of the interaction of employees ( E2E ), Enterprise 2.0 means an environment in which communication processes employed circumvent the existing formal and bureaucratic structures enabling new forms of cooperation and collaboration and thus the sharing of knowledge and expertise, which plays an important role in the formation of intelligent organizations. In this way, possible shortcomings in the existing structure are overcome, the creation of informal groups is established through social interactions that are formed on the basis of performance of everyday tasks, and not on the basis of formal manager`s task [3].

A successful example of the corporate social network Yammer is Microsoft product, which is implemented as a platform in the Deloitte company [20]. Yammer offers integration with the well-known Office package to enable the sharing of materials in different formats. Another example is the in-house developed solution for the IBM [14] called ThikPlace, back in 2005, with the aim of sharing knowledge and ideas when, within the period of two years 18,000 new approaches to solving of daily work problems were generated, while about 350 ideas were applied in practice, earning about \$ 500 million. [14]

BeeHive [7] is designed as a Web site based on the user profile and share blogs. This system was implemented in 2010 and has provided significant benefits in terms of building social capital and knowledge transfer within the multinational companies.

Cooler [6] is an in-house built platform to Hewlett-Packard Company, which has properties of aggregated social communication channels such as blogs, wikis, instant messaging, etc. The given platform enables the analysis of communication.

\section{Metrics analysis of online social network}

The massive use of social applications brings opportunities of monitoring actual communications with the aim of measuring the mutual staff relations. It includes social application platforms within companies and corporations, enabling new sources of information of interest to human resource managers in terms of recognizing hidden relations and employee relationships [21]. Techniques and methods of investigation of corporate social networks are in the area of social network analysis, which aims to determine the characteristics of the actors in the network and their mutual relations.

The measurement and analysis of social networks (hereinafter SNA) is an approach to research which sees the analyzed network as a graph, and is grounded in the field of mathematics called the Graph theory. A graph consists of a set of nodes (vertex) and a number of branches (edge) with a quality that each branch has two (it is not obligatory for them to be different) nodes. The Graph is represented as a tuple $G=(V, E)$ where $V$ is the set of nodes, and $E$ is the set of edges [3]. Research and analysis of social networks is made in a manner that the nodes are people or sometimes groups of people, while the branches represent a kind of social interaction among the people, such as friendship. [16]

This approach which is seen as a complex network graph allows for the identification of hidden relationships and connections, provides visualization and insight into the structure, allows for measurement, testing of connectivity and optimization of elements within the network. The Graph theory allows for the prediction, organization, a different view of the structure of the network or its components, tracking the spread of useful information, but also of harmful components such as viruses in computer networks. 
Depending on a manner in which the network is observed the analysis of the whole network (macro analysis), of a certain part (mezo analysis) or ego network analysis (micro analysis) can be distinguished. Ego network refers to a network node with which the analyzed node is connected. The network analysis is done with the pre-defined metrics and they is divided into three categories [22]:

a) monadic metric-calculation of individual nodes,

b) dyadic-metric computations for a pair of nodes,

c) metric-graph computations for a graph instance network density.

Here is an example method in the field of monadic metrics: degree of the node, normalized node degree, closeness node, the centrality of the node, connections between, Jordan centrality, ranking pages and so on.

For each of these there is a mathematical definition of the metric. In this paper, the calculation of the metric used is open source software with built-in algorithms to calculate the above metrics. Moreover, it discusses the possibility of using some metric methods of analysis of social networks with the objectives of selection and identification of appropriate staff within the corporation.

The following connectons were used: degree of the node, HITS analysis, connections between identification of hidden clusters and the density and cohesion of the network.

\section{Description of research}

One of the tasks of the human resource manager is shifting and employment of new labour within the corporation. Applying online SNA method in the process of identifying the type of staff is possible if the corporation has implemented some form of online social applicative platforms. In this way it is possible for corporations, in addition to the existing data, to include the social component of employees if it is relevant to their choice [17].

The SNA techniques are suitable for detecting hidden groups that may exist as a result of physical boundaries (different geographical locations) and organizational structure ( rigid hierarchical structure or different domain expertise). In addition, these techniques are suitable for the detection of individual leaders in the network or the employees that connect multiple organizational units into a harmonious whole. Some possible research objectives of corporate social networks are [10]:

- development and improvement of social networks with the aim of sharing knowledge and expertise,

- detecting differences between formal and social structure of employees of the corporation for the purpose of restructuring,

- discovering 'hidden' leaders,

- detecting bottlenecks of information flow,

- creating of a community instead of teams in the execution of tasks,

- implementation of education and training through Enterprise 2.0 platform,

- and other.

This paper explores the example of predefined organizational units to reach a number of employees who fit the required profile. The main weakness of SNA techniques is their fundamental quantitative parameters of nodes in the network. In this paper we present an approach where, in addition to quantitative, additional parameters are introduced in combination with SNA method to enable HR managers to perform complex queries against a corporate social network.

The approach is based on multiple filtering data in order to enhance query performance for a large number of records, and a more efficient use of IT resources. The paper used the JUNG Java libraries [12] designed to explore the data set using the method of analysis of social networks, which include research algorithms.

\section{Review of the concept of extended sna analysis}

The introduction of new dimensions in the SNA is necessary for two reasons. The first reason is a more precise data filtering and getting to new information, obtained through classical SNA analysis [13]. Another rea- 
son lies in the absence of JUNG framework, which requires loading of test data sets in the internal memory, which in case of large amounts of data can be a significant problem.

A total of six defined dimensions are involved in the analysis process. The framework represents the employees, and is formed on the basis of actual communication while dimensions are filters that help in identifying the required grades.

The basic dimensions of the extended SNA is an organizational unit defining the scope of the analysis in relation to the organizational structure of the corporation. This means that we determine the subset of social communication in relation to the organizational structure of the corporation where there is a possibility of simultaneous analysis of multiple organizational units.

Time as a dimension of analysis can be given as an arbitrary date range or a period of execution of a specific project. In this way it is possible to determine the characteristics of the nodes / employees of the port specified period of time, for example. It may be a period of crisis for the execution of a project. In this way, we can identify employees who are willing to take responsibility, to share knowledge and provide expertise to other employees. It is possible to set the time step in which the analysis will be carried out where steps may be days, months, and years.

Location is important as a dimension if a corporation that is distributed over a wider geographical area. In this way we obtain information on communications between employees in different locations.

The dimension of personal data is a classic collection of data that organizations keep on their employees. This dimension is of utmost importance in identifying employees. It is through this dimension that human resource managers define the characteristics that the required employee must fulfill.

The dimension context of the message refers to the message content to be transferred. In our case, the context of the message is determined by its title, which must contain the name of the project when it is assumed that the content of messages is related to the project from the title of the message.

The dimension type of communication refers to communication channels and is defined as e-mail or blog post system. The simulation enables a simultaneous analysis of social interaction in both communication channels.

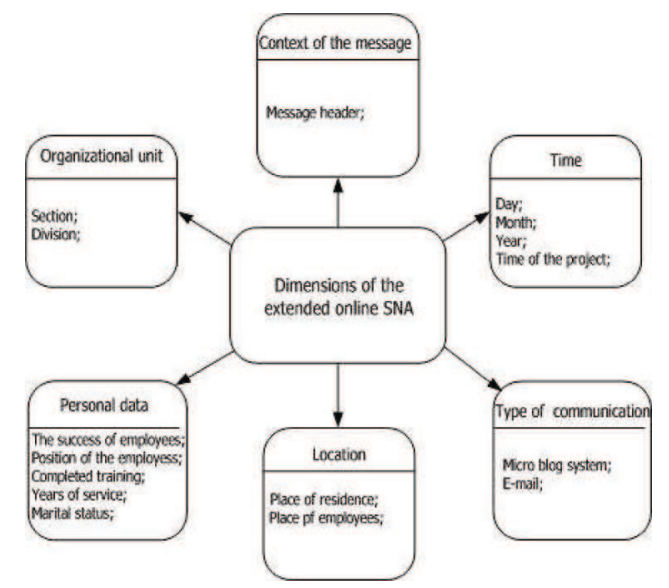

Figure 1. Dimensions of SNA

\section{SNA features applications}

For demonstration purposes, the functionality extended SNA developed an application that simulates a corporate social network. The application can be classified as type blog system that uses posts and e-mail messaging. All communications, profiles and data in the study were created by typing random strings in the database. The selection is performed in files:names.txt. surname.txt, cities.txt, LevelofProfessionalDegre.txt. 
etc. containing arrays of strings. By random selection of a string from the mentioned files, the object employee is established and entered into the database. In the same way, objects Training and projects are established whereas in the next stage objects profileEmployee would be created. After completing the database it is possible to run the application.

The application consists of two main modules: administrator and user. It is possible to log on as an administrator or as a user application. Administrator has the right to view the user profiles and performance of the extended SNA. In the admin module the user selects the parameters needed to execute the extended SNA. The selection of the parameters is carried out in several steps, where each step corresponds to one dimension of the SNA that was previously mentioned herein. After setting the parameters and selecting the presentation, the results will be displayed in the form of charts and graphs, and the tabulation of results.

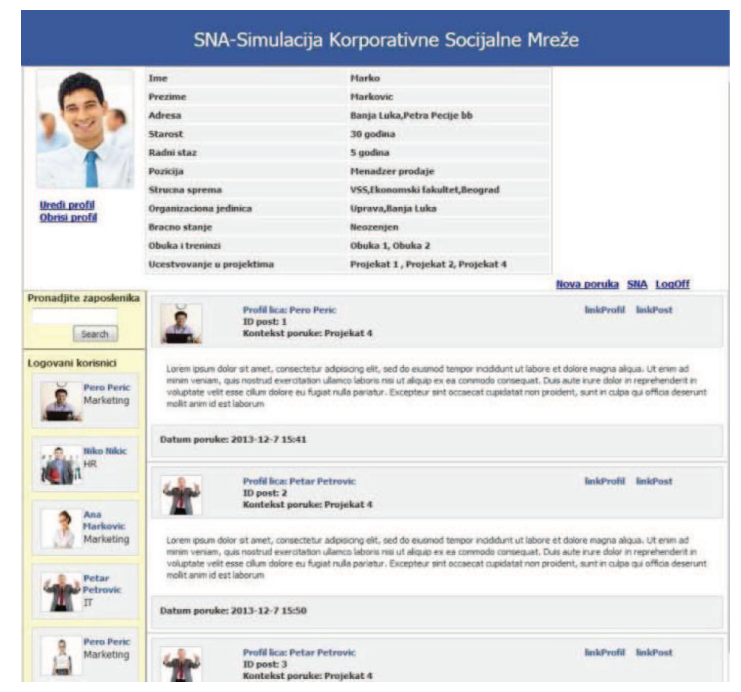

Figure 2. Employee profile in SNA application

\section{Used technologies}

The application is made to the model -view- controler software patterns. To build the model, components are written in DAO and DTO classes that communicate with the MySQL database. Within the model components the JUNG ( Java Universal Network / Graph Framework) framework [12] was used, which enables the creation and visualization of objects graphs. Jungle is an open source library that enables the development of Web and desktop application for exploring and analyzing data sets of its theoretical basis found in the area of mathematics called the Graph theory. The basic phases of the data sets are importing, modelling, analysis, visualization and data filtering. For each of these phases the JUNG package provides classes that implement the interface methods and algorithms from the field of graph theory, data mining and analysis of social networks. For the development of component controler JavaServlet technology was used, while the presentation used JSP, jQuery and java script d3js [11] frameworks.

\section{Description of the implemented SNA methods}

\section{Degree of the node}

This analysis gives us a quantitative sociological data on social ties of individual employees. The sociological aspect is reflected in the relationship between input and output connections. Employees with the highest output connections can be hidden leaders in the organization. According to the survey [18], 50\% $-70 \%$ of knowledge required to perform daily tasks employees receive from their colleagues in the region. The node with the highest ratio of output and input level shall be determined by the employees (Authority) who are the initiators of communication and knowledge sharing across the network and the audience (Hubs) that receive the information. The input level is the ratio of the amount of input and total communication network while the output stage is the ratio of the amount of output and total communication. Determining of the 
degree of the node for the purpose of identifying the leaders in the network can play an important role if it determines a "critical" days of the project. The critical days means the period in which difficulties appear or unforeseen circumstances in the project emerge. Figure 3 shows the movement of the degree level of randomly selected employees for the duration of the project and for two different communication channels. If the peak of the degree of the node level coincides with the days when the project was in critical stages of implementation, it is likely that it involves a hidden leader from whom other employees are seeking assistance and expertise. Figure 4 shows the degree of node stage of the three top-ranked employees where the output follows the input stage of the employee by day.

In Figure 5, data are visualized and analyzed in the form of the graph in which nodes are employees who meet the requirements defined in the dimensions of SNA. The graph is directed and in it you can see trends and directions of information within the study area. During the net visualization, input and output degree of node are not concidered, whereas the position of node in the graph is determined by the number of connected neighboring nodes and weight value of branchies amounts to1 for any number of realised communications.

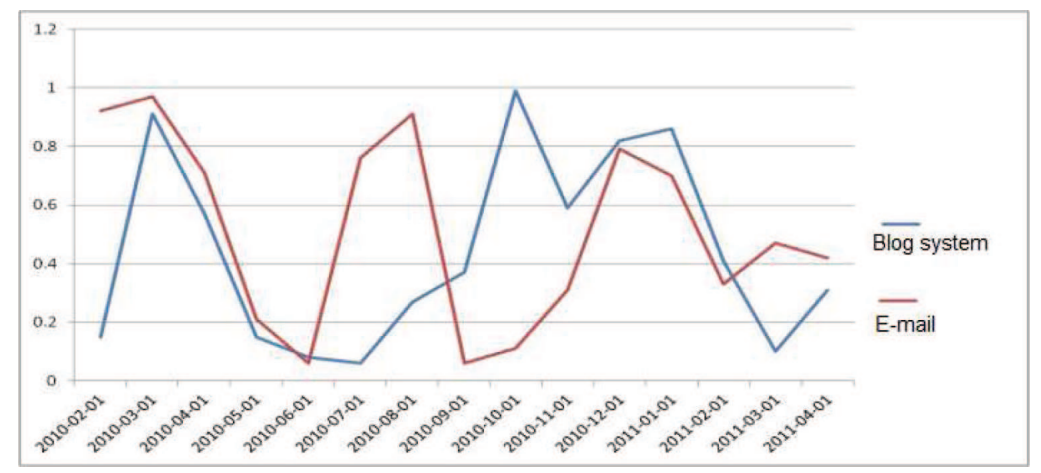

Figure 3. Display of two degrees of nodes for different communication channels

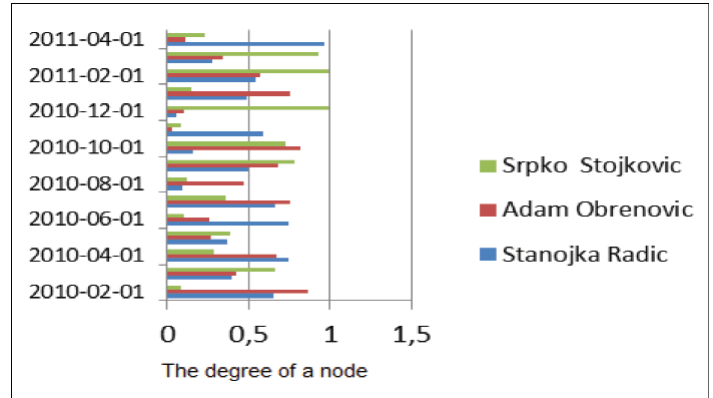

Figure 4. Gantt value of the degree of a node level of the top-ranked employees

By measuring the degree of a node employees we obtain the means by which we identify nodes that have the highest level of communication within the corporation. These employees have specific knowledge of the importance of carrying out everyday tasks.

If the human resource manager needs a worker with specific skills and knowledge, it is advisable to conduct this type of analysis.

\section{HITS analysis}

The HITS analysis is based on the discovery of the best 'recipient' and the best 'sender' ('Hub and Authority") information. The Authority estimates the value of the node importance as a source of information, while the Hub provides an assessment of the significance of the value of the node as a gatherer of information 
[16]. the value of Hub factors is higher if a node receives information from high-ranking Authority nodes [19]. Employees identified as sources of information tend to transfer their knowledge, while employees identified as gatherers of information / knowledge/ tend to learn and absorb new information.

The HITS analysis may be particularly interesting for human resource managers if they strive to identify a prospective employee who shows characteristics of constant learning and development. A possible application scenario for the HITS analysis would be the identification of young, promising workers with the aim of setting up a post which requires continuous learning and training where the requested person has developed contacts with leading experts within the corporation. This task can be performed by ranking the value hub of factors employed and the inclusion of the extended SNA, by defining the selection of data such as age, location, qualification, etc..

\section{Analysis of betweennes}

Analysis of betweenness indicates the number of shortest paths from all vertices to all other peaks that pass through the node analyzed [5]. These are bridges in the network, the employees who connect different clusters. This type of analysis is particularly important in identifying leaders in the network and employees who are of significant importance to the corporation on account of their hard work and knowledge of the associated group as a whole [5]. It is based on the calculation demonstrating the level of role of a particular node in terms of connecting two or more groups within the network. The corporation is most commonly employed to work with the departments for different purposes e.g. employees who coordinate the work of the department of marketing and development, etc. [17].

It is a measure of the burden and significance of the observed node. It shows that employees are more important for the exchange of information between other employees. It is also used as data indicating where the network communication would fall apart if particular nodes were elininated, that is, which employees would be disconnected if part of nodes/ employees is removed.

The application allows examination of data for analyzing connections between employees, with predefined dimensions search. Figure 5 shows the generated graph from which it is clear that there are three employees with the highest level of connections between them.

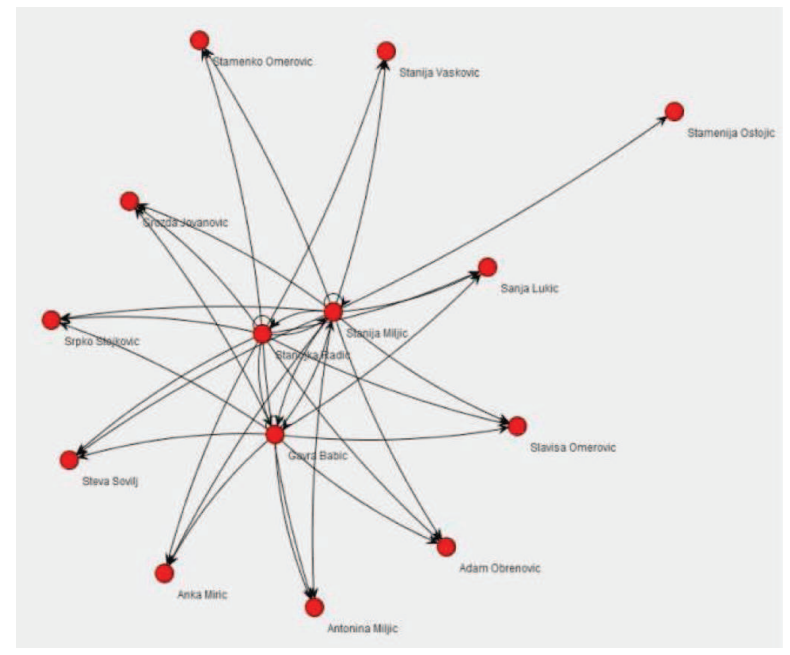

Figure 5. View graph of employees

Betweenness may be relevant to human resource managers in terms of identification of employees who have the ability to transfer information and coordinate activities among various departments, clusters and groups. These employees are characterized by the ability to coordinate activities between organizational units and by expertise in many different areas. If it is necessary to identify employees with the ability to coordinate and manage teams, it is advisable to conduct an analysis of betweenness. 


\section{Identification of hidden cluster}

Clusters are closely related subgroups within the organization network. The algorithm identifies clusters as input parameters using size density and number of branches per node. In this way it is possible to discover the social structure of the organization, which can significantly differ from the formal structures. An interesting approach is presented in the study [1] where the actual network structure is obtained on the basis of the LDAP directory and the degree of difference with overlapping two graphs, structural and social. On the basis of the degree of diversity, managers make decisions about the need for changing the structure of the organization. Identification of cluster has an important role in the context of the change process and the restructuring of the organization and also, very important information may be obtained by detecting the leading node within each cluster.

Ranking and discovering of the leading node in the cluster is done by measuring the output of the input level of the nodes in the cluster. In this way, we can identify employees who are informal leaders forming clusters. This information is important for human resource managers if they are trying to identify employees with the capabilities of team leaders or department leaders.

Table 1: Identified clusters and leaders of clusters

\begin{tabular}{|l|c|c|c|}
\hline Clusters & $\boldsymbol{\Sigma}$ & Employee ID & Leader ID \\
\hline Cluster 1 & 12 & $2,15,23,20,11,49,52,38,33,34,14,5$ & 15 \\
\hline Cluster 2 & 8 & $17,19,8,44,32,39,50,11$ & 44 \\
\hline Cluster 3 & 5 & $26,7,3,13,31$ & 7 \\
\hline
\end{tabular}

\section{Density and cohesion}

Unlike previous measures that address the ego network, i.e., the individual employee, density and cohesion are parameters that tell us about the state of the entire network [22]. Density is a parameter that tells us about the relationship between the number of existing and possible branches in the network. This size is in the range from $0 \%$ to $100 \%$. Researched networks have a size from $5 \%$ to $30 \%$ for frequently used and connected network, although this value may vary depending on the size and purpose of the network. The measure of cohesion refers to the measurement of the maximum distance between two nodes in the network. Preferably, the network has a lower degree of cohesion because it implies shorter distance for submissions. For example, density of 3 means that any employee may submit the information to the other without having information pass through node 3 .

Measures of density and cohesion play an important role in the analysis of individual organizational units. From the standpoint of management, if analyzed network has no specific density or if it has a density less than $5 \%$ [2], the results of previously performed analysis can be safely treated as valid. This is especially the case if the communication performed by the analysis was done in a short period of time, or if the application in use is in social terms fully operational within a corporation. It can be said that the measure of density is proportional to the credibility of the results obtained by the extended SNA analysis. In this case, the density was $15 \%$.

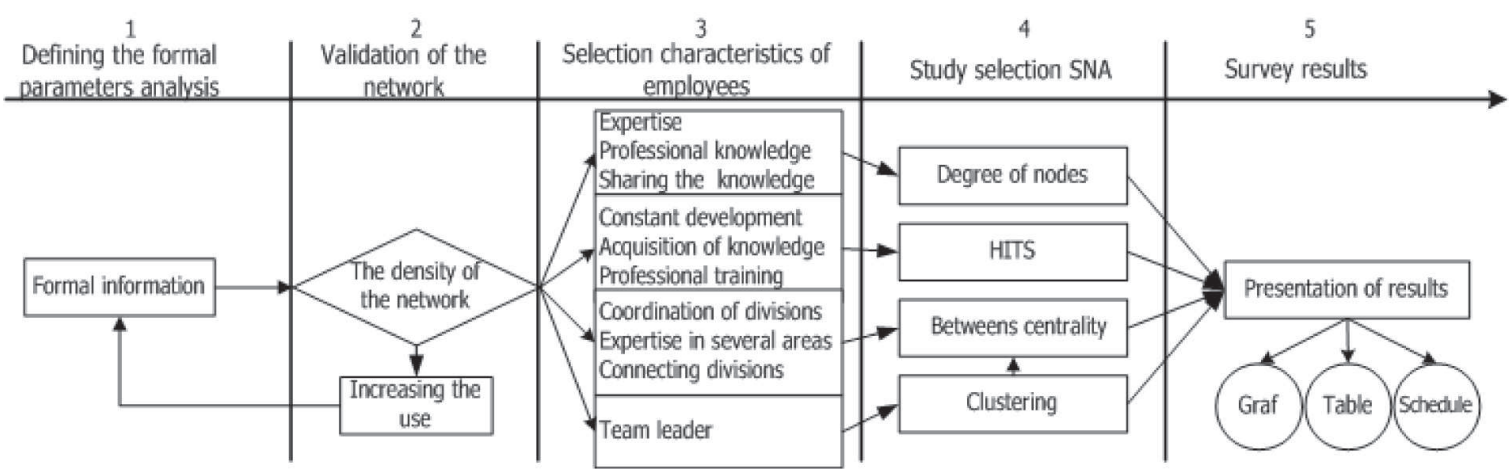

Figure 6: Flow of extended SNA 


\title{
13. Closing remarks
}

The above method of the analysis of social communication allows us to discover the profile of an employee who meets the requirements of human resource managers. In addition to formal information about employees, when making selection, managers also have social factors at their disposal. Staff selecting follows the steps shown in Figure 6.

\begin{abstract}
In the first step, the HR manager defines the formal requirements that the employee should satisfy. Formal requirements are related to the personal data of employees, location and organizational affiliation. After that, based on testing network communication, density factor for the validation of the reliability of the results is measured and if it is greater than $5 \%$, it goes to the next step. If the density of the network is less than $5 \%$, the human resource managers must take actions in order for the use of social applications to become massive within the corporation. This can be achieved by adopting special procedures or conducting internal marketing campaigns to use social applications in their daily work [2].
\end{abstract}

The next step is a selection of social characteristics that employees must possess. In our case, there are four groups of characteristics of employees. The first group of characteristics is selected if an employee is sought who is an expert in its domain, the second group is selected if an employee is needed who is prepared to invest in their own development efforts, the third group is selected in case a coordinator is required between multiple organizational units while the fourth group is selected if the team leader is required.

Finaly, the method of analysis is selected automaticly depending on the characteristics of a selected employee. A format presentation of the results depends on the selected SNA method and is given in the form of charts, graphs and spreadsheets ranking.

In this paper the possibility of using the social network analysis in the selection process and selection of employees with a view to fill the jobs from existing resources or the replacement of employees in the existing workplace is discussed. An application is developed that aims to simulate the communication network of employees, generating a profile of employees and their mutual communication. On the basis of these data several analyzes were performed and their possible application in terms of selection of employees is explained. A new concept, the so-called extended SNA, was introduced to application where, in addition to the quantitative approach to research networks also, formal parameters related to the personal data of users as well as contexts that are related to the content of messages that users exchange, were introduced. This approach allows for the introduction of additional dimensions in the process of analyzing online corporate social networks.

The inclusion of elements of business intelligence and semantic analyses represent the future challenges in the development of application mentioned in this paper.

In the next stage of research, introduction of weight coefficients for analysis of nodes in network is planned.

Further work is focused in two directions. In the first case it is necessary to test the operation of the application in a real environment, which should serve as a Social Application Platform in the organization and eliminate technical deficiencies and improve the operation of the application. Subsequently, on the basis of actual communication between employees, it is necessary to test the approach of the extended SNA by examining the validity of the results.

\section{REFERENCES:}

[1] Antonio Zilli, Robert Laubacher, Peter A., (2006), E-mail May Not Reflect The Social Network: Gloor NAACSOS Conference, on June 22 - 23, Notre Dame IN, North American Association for Computational Social and Organizational Science

[2] Awolusi F., (2012), The Impact of Social Networking Sites on Workplace Productivity, The Journal of Technology, Management, and Applied Engineering, Vol.28, No.1.

[3] K. Bogart, C. Stein, Drysdale RL, (2005), Discrete Mathematics for Computer Science, Key College Publishing, Emeryville, Canada. 
[4] Boyd, Danah M., Ellison, Nicole B. (2007) Social Network Sites: Definition, History, and Scholarship, Journal of Computer-Mediated Communication.

[5] H. Bruce, Claire R., (2010), Social network analysis and the evaluation of leadership networks, The Leadership Quarterly, 21, pp. 600-619.

[6] Brzozowski M., (2009), Watercooler: exploring an organization through enterprise social media, Proceedings of the ACM 2009 International Conference on Supporting Group Work, pp. 219-228, ACM New York.

[7] S. Charles, Joan MD, Nicole BE, Cliff L., ( 2012), Bowling Online: Social Networking and Social Capital within the Organization, Proceedings of the Fourth International Conference on Communities and Technologies, pp. 245-254.

[8] Chelmis C, Prasanna P., ( 2011), Social Networking Analysis: A State of the Art and the Effect of Semantics in Social Computing ( Socialcam ), IEEE Third International Conference, MIT, Boston, USA, IEEE.

[9] Chelmis, C. Sorathia, and V. Prasanna, V., ( 2012), Enterprise Wisdom Captured Socially, page In 12281235, Istanbul, Turkey.

[10] Efraim T., B. Narasimha, Ting - Peng Liang, (2011), Enterprise Social Networking: Opportunities, Adoption, and Risk Mitigation, Journal of Organizational Computing and Electronic Commerce, 21:3, 202-220

[11] http://d3js.org/, accessed on 11/12/2013.

[12] http://jung.sourceforge.net/, accessed on 11/12/2013.

[13]L. Humski, Striga D., Podobnik V., B. Vrdoljak, Banek M., (2013), Skočir Building implicit corporate social network, ZITelecommunications ( ConTEL ), 12th International Conference in Zagreb

[14] A. Majchrzak, Cherbakov L., Ives, B. (2009) Harnessing the power of the crowds with corporate social networking tools: How IBM does it? MIS Quarterly Executive, Vol. 8 Issue 2, P103 Academic Journal.

[15] AP McAfee, (2009), Enterprise 2.0: New Collaborative Tools for Your Organization's Toughest Challenges, Harvard Business School Press, Boston, MA, USA.

[16] Newman MEJ, (2010), Networks: an Introduction, Oxford University Press, Oxford, NY

[17] S. Paulus, Pryzemyslav K., (2010), Social Network Analysis in Corporate Management, Advances in Intelligent and Soft Computing, Volume 80

[18] S. Phulari, Khamitkar Dr.SD, Deshmuk NK, PU Bhalchandra, SN Lokhande, ARShinde, (2010 ), Understanding Formulation of Social Capital in Online Social Network Sites ( SNS), International Journal of Computer Science Issues, IJCSI, Vol. 7, Issue 1, no. 3

[19] K. Pranam, Tim F., Yelena Y., Y. Yaacov, L. Kelly, Stephen P., Jen H., (2007 ), On the Structure, Properties and Utility of Internal Corporate Blogs, Proceedings of the International Conference on Weblogs and Social Media ( ICWSM 2007).

[20] G. Reime, Scifleet P., (2012), Enterprise Social Networking in Knowledge-Intensive Work Practices: A Case Study in the Professional Service Firm, 23rd Australasian Conference on Information Systems Enterprise Social Networking in Knowledge Work.

[21] S. Sovilj, Vasković V., (2013), Analysis of corporate social nerworks using the JUNG framework, InfoM 48, 15-20, UDC: 004.7:681.3

[22] Wasserman, Stanley and Faust, Katherine., 1994th Social Network Analysis: Methods and Applications. Cambridge: Cambridge University 


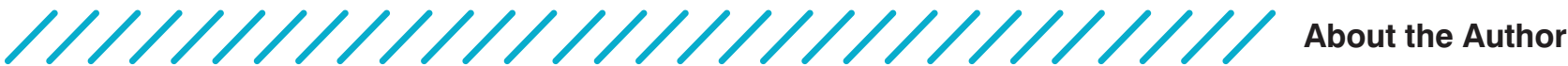

\section{Slaviša Sovilj \\ Ministry of Internal Affairs of the Republic of Srpska, Bosnia and Herzegovina sovabass@yahoo.com}

Slaviša Sovilj was born in Split in 1979, lives at Gradiska $(\mathrm{BiH})$ and is currently employed in the Ministry of Internal Affairs in Banja Luka. He has graduated from the Military Academy in Belgrade in 2002, majoring in the Electronic Warfare-Telecommunications programme. He got his Masters degree at the Faculty of Organizational Sciences, University of Belgrade in 2010 on the Electronic business programme. He also got certificates for a successful completion of basic and advanced courses for Java programming at the Faculty of Electrical Engineering in Banja Luka.

His areas of interest are programming of network and Web applications, data mining, social network and big data analysis.

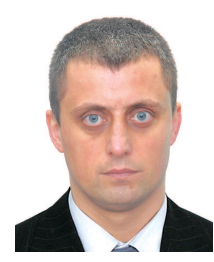

Vojkan Vasković University of Belgrade, Faculty of Organizational Sciences, the Republic of Serbia vojkan.vaskovic@fon.bg.ac.rs

Vojkan Vaskoviæ is assistant professor at the and professor at Belgrade Business School. Vaskovic received his PhD at the Faculty of Organizational Sciences, University of Belgrade (1997). His main research interests include electronic business, e-banking, social networking, e-Government and multimedia.

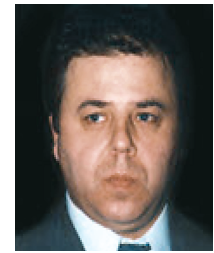

